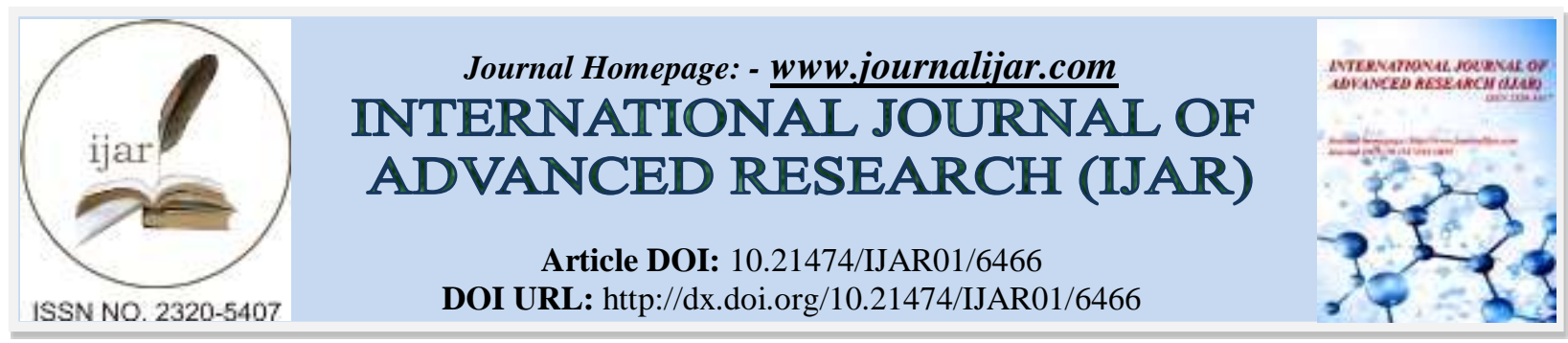

RESEARCH ARTICLE

\title{
INVERSION UTERINE NON PUERPERALE.
}

Mounsef Mahaouchi $^{1,2}$, Mohammed Karam Saoud ${ }^{1}$, Najoua Douzi ${ }^{1}$, Siham Boumhaoud $^{1}$, Nisrine Mamouni ${ }^{1}$, Sanaa Errarhay ${ }^{1}$, Chahrazad Bouchikhi ${ }^{1}$ and Banani Abdelaziz ${ }^{1}$.

1. Service gynécologie-obstétrique I, CHU HASSAN II de Fès, Maroc.

2. Auteur correspondant : Mounsef Mahaouchi, service gynécologie-obstétrique I, CHU HASSAN II de Fès, Maroc.

\section{Manuscript Info}

\section{Manuscript History}

Received: 11 December 2017

Final Accepted: 13 January 2018

Published: February 2018

\section{Key words:-}

uterine inversion; Non-puerperal;

conservative treatment;

submucosal fibroma.

\section{Abstract}

Uterine inversion is a very rare gynecological complication that represents a real emergency. It is classically described in serious obstetric complications. In a non-puerperal situation, it is most often due to the development of a process that is benign or malignant at the fundic level of the uterus. Nevertheless, cases of idiopathic uterine inversion have been reported in the literature. We report here the case of a patient aged 35 in our training for metrorrhagia in an anemic patient. The examination objectified an acute uterine inversion due to a fundic process. The surgical management was done by a double approach: laparotomy and vaginal.

Copy Right, IJAR, 2018, All rights reserved.

\section{Introduction:-}

L'inversion utérine est une entité rare, encore plus rare sur un utérus non puerpéral $[1 ; 2]$. Elle est définie par un retournement de l'utérus en doigt de gant. Elle est due au développement d'un processus bénin ou malin au niveau fundique, le plus fréquemment il s'agit d'un fibrome sous muqueux [3;4]. Cette situation peu commune peut donner lieu à un retard de diagnostic et retarde la prise en charge chirurgicale qui représente une véritable urgence.

\section{Patient et observation :-}

Il s'agit d'une patiente âgée de 35ans, multipare, sans antécédents pathologiques notables, admise aux urgences de notre formation pour métrorragies évoluant depuis cinq jours avec notion de douleur pelvienne aigue expulsive avec protrusion d'une masse à travers la vulve.

L'examen clinique trouve une patiente en assez bon état général, instable sur le plan hémodynamique, conjonctives décolorées, avec une masse vulvaire à l'inspection périnéale sans identification du fond utérin aux touchers pelviens, faisant suspecter en premier une inversion utérine (figure 1)

L'échographie sus pubienne confirme le diagnostic d'inversion utérine (figure 2)

Une tentative de réintégration utérine (Manœuvre de Johnson) a été sans succès.

NFS avec $5 \mathrm{~g} / \mathrm{dl}$ d'hémoglobine, elle fut transfusée par 4 culots globulaires et 4 plasmas frais congelés avant l'acte opératoire 
Un double abord par voie vaginale et abdominale (figure 3) permettant la réalisation d'une myomectomie par voie vaginale (figure 4) suivie d'une colpotomie postérieur avec réduction de l'inversion puis une plicature des ligaments rond et une ligature section des trompes.

L’histologie était en faveur d'un léiomyome utérin

\section{Discussion:-}

L'inversion utérine non puerpérale est extrêmement rare. Il n'existe aucune donnée épidémiologique vu que les cas publiés sont des cas sporadiques.

L'inversion utérine non puerpérale est due le plus souvent au développement de myomes sous séreux au niveau fundique. Cette inversion est expliquée par l'atrophie et l'affaiblissement de la paroi utérine crée par le myome fundique, ceci est d'autant plus marqué que le myome est large. Associée a ceci les contractions musculaires du muscle utérin stimuler par le prolapsus de la tumeur dans la cavité utérine.

Une classification des inversions utérines a été décrite [5] :

- $\quad 1^{\text {er }}$ degré : l'inversion utérine est intra-utérine ou incomplète. Le fond reste dans la cavité.

- $\quad 2^{\text {ème }}$ degré : inversion complète du fond utérin à travers le col utérin.

- $3^{\text {ème }}$ degré : inversion complète du fond utérin qui est extériorisé à travers la vulve.

- $4^{\text {ème }}$ degré : participation des parois vaginales au retournement.

Notre patiente présentée une inversion du $3^{\text {ème }}$ degré.

L'étiologie la plus fréquente (70-85\%) est le myome sous-muqueux qui était le cas de notre patiente. Takano et al [6] Ont rapportés 88 cas d'inversion utérine non puerpérale. $92 \%$ des cas était du a des tumeurs utérines dont $20 \%$ des cas était des tumeurs malignes. Parmi ces tumeurs malignes on retrouve les leiomyosacromes utérins, les rhabdomyosarcomes, les sarcomes du stroma endométrial, moins fréquents les inversions sur cancer de l'endomètre [7] ou sur tumeur mullérienne mixte [8].

Notre cas était un léiomyome utérin

Le diagnostic des inversions utérines est facilement posé par la clinique lors des stades 2 à 4 . Tandis que pour le stade 1 le recours a des moyens d'imageries s'avère nécessaire. L'échographie en coupe transversal montre un utérus avec un aspect en cible avec un fond hyperéchogène entouré d'un bord hypoéchogène qui représente le liquide situé dans l'espace entre le fond utérin et les parois du vagin. En coupe sagittale on retrouve l'aspect d'une image en miroir d'un utérus normal. L'IRM s'avéré être un moyen diagnostic très utile. Lewin et al ont rapporté qu'en séquences pondérées $\mathrm{T} 2$, une cavité utérine en forme de $\mathrm{U}$ avec un fond épaissi et inversé en coupe sagittale associé à un aspect d'œil de taureau sur une coupe axiale, était très évocateur d'une inversion utérine [9]. Notre patiente n'a bénéficié d'aucun examen complémentaire vu que le diagnostic a été facilement retenu sur la clinique et que son état hémodynamique ne pouvait laisser tarder la prise en charge.

La principale complication lors des inversions utérines aigue est le risque hémorragique qui peut mettre en jeu le pronostic maternel et qui doit être bien géré avant d'entamer un geste chirurgical [10]. Notre patiente était admise avec $5 \mathrm{~g} / \mathrm{dl}$ d'hémoglobine, elle fut transfusée par 4 culots globulaires et 4 plasmas frais congelés avant l'acte opératoire.

Le pronostic des inversions utérines dépend du stade initial du diagnostic et le stade de l'inversion [11].

Plusieurs prises en charge ont été décrites dans la littérature : un traitement conservateur quand la réduction de l'inversion utérine est possible, principalement en cas d'inversion utérine du $1^{\mathrm{er}}$ ou du $2^{\text {ème }}$ degré. Le traitement radical est privilégié en l'absence de désir de grossesse, en cas de pathologie maligne et pratiquement dans tout les cas d'inversion du $3^{\text {ème }}$ et $4^{\text {ème }}$ degré. Selon Auber er al le double abord par voie coelioscopique et vaginal semble être une bonne alternative pour confirmer le diagnostic et dévasculariser l'utérus à l' origine du pédicule utérin. Pour notre patiente la cœlioscopie n'était pas disponible en situation d'urgence, donc on a réalisé un abord par laparotomie et par voie vaginal. On a opté pour une attitude conservatrice vu le désire l'âge de la patiente (figure5). 


\section{Conclusion:-}

L'inversion utérine non puerpérale est une affection très inhabituelle dont la plupart des gynécologues ne rencontreront jamais, et qui doit être gérée sur la base d'une expérience antérieure minime ou inexistante. Malgré que les causes malignes sont rares il faut qu'elle soit toujours envisagé et réalisé des biopsies préopératoire pour mieux orienté l'acte chirurgical.

\section{Conflits d'intérêts :-}

Les auteurs ne déclarent aucun conflit d'intérêts

\section{Contributions des auteurs :-}

Mounsef Mahaouchi : prise en charge diagnostique de la patiente, contribution à la prise en charge thérapeutique. Mohammed Karam Saoud : contribution à la prise en charge thérapeutique, contribution à la recherche bibliographique et rédaction de l'article. Najoua douzi, Siham Boumhaoud, Nisrine Mamouni, Sanaa Errarhay, Chahrazad Bouchikhi, Banani Abdelaziz : contribution dans la prise en charge diagnostique et thérapeutique

\section{Tableaux et figures}

Figure1

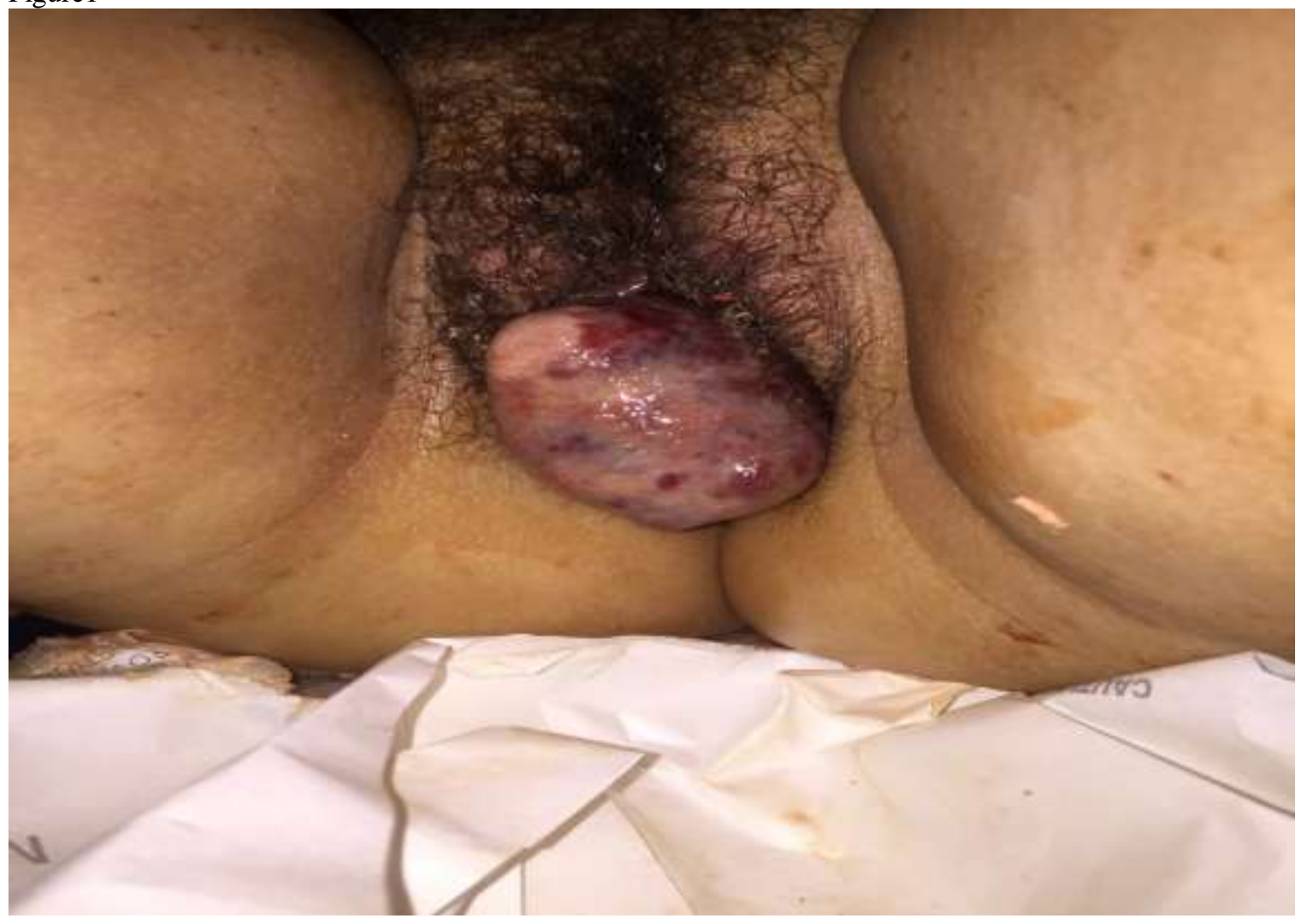


Figure 2:-

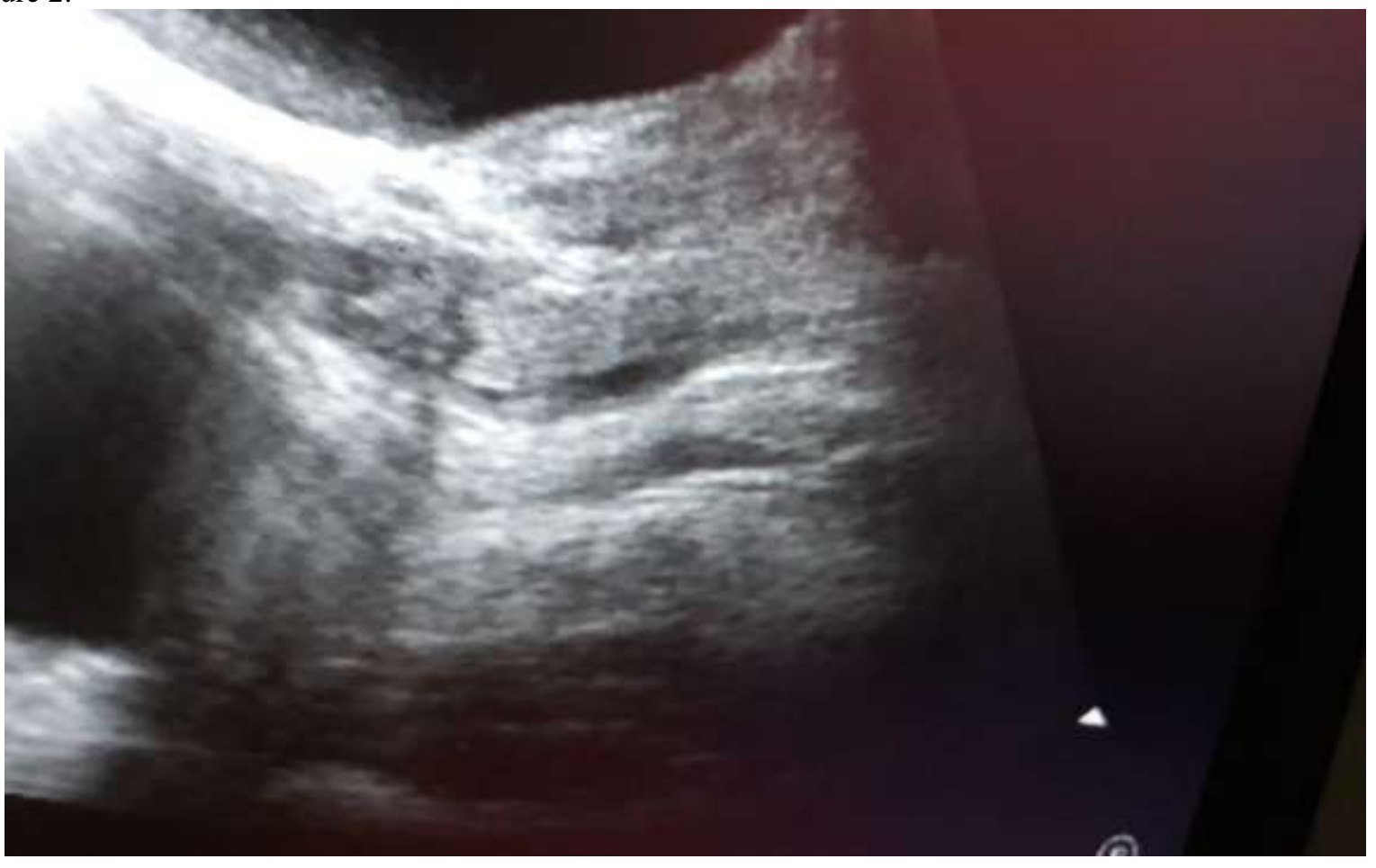

Figure 3:-

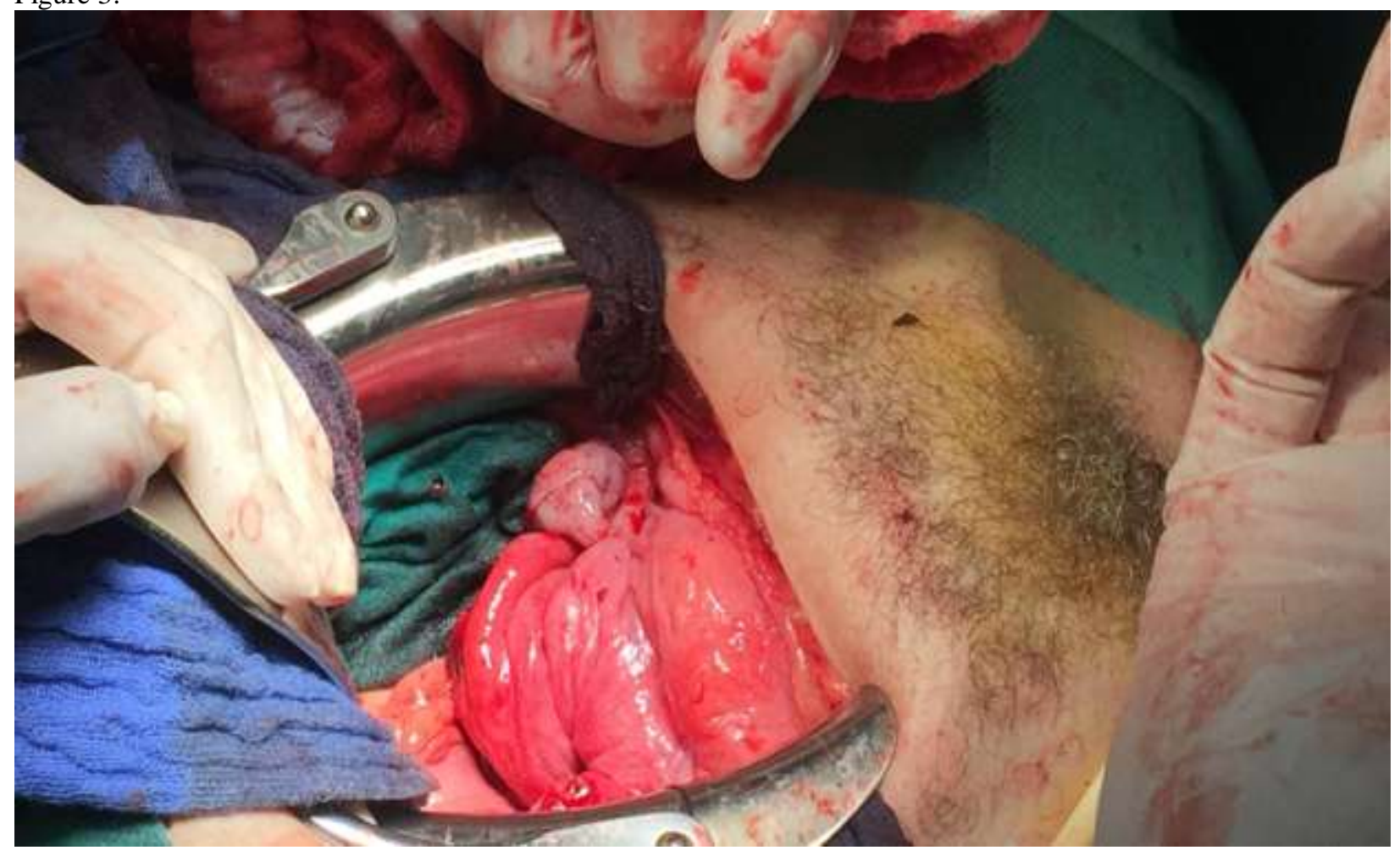


Figure 4 :

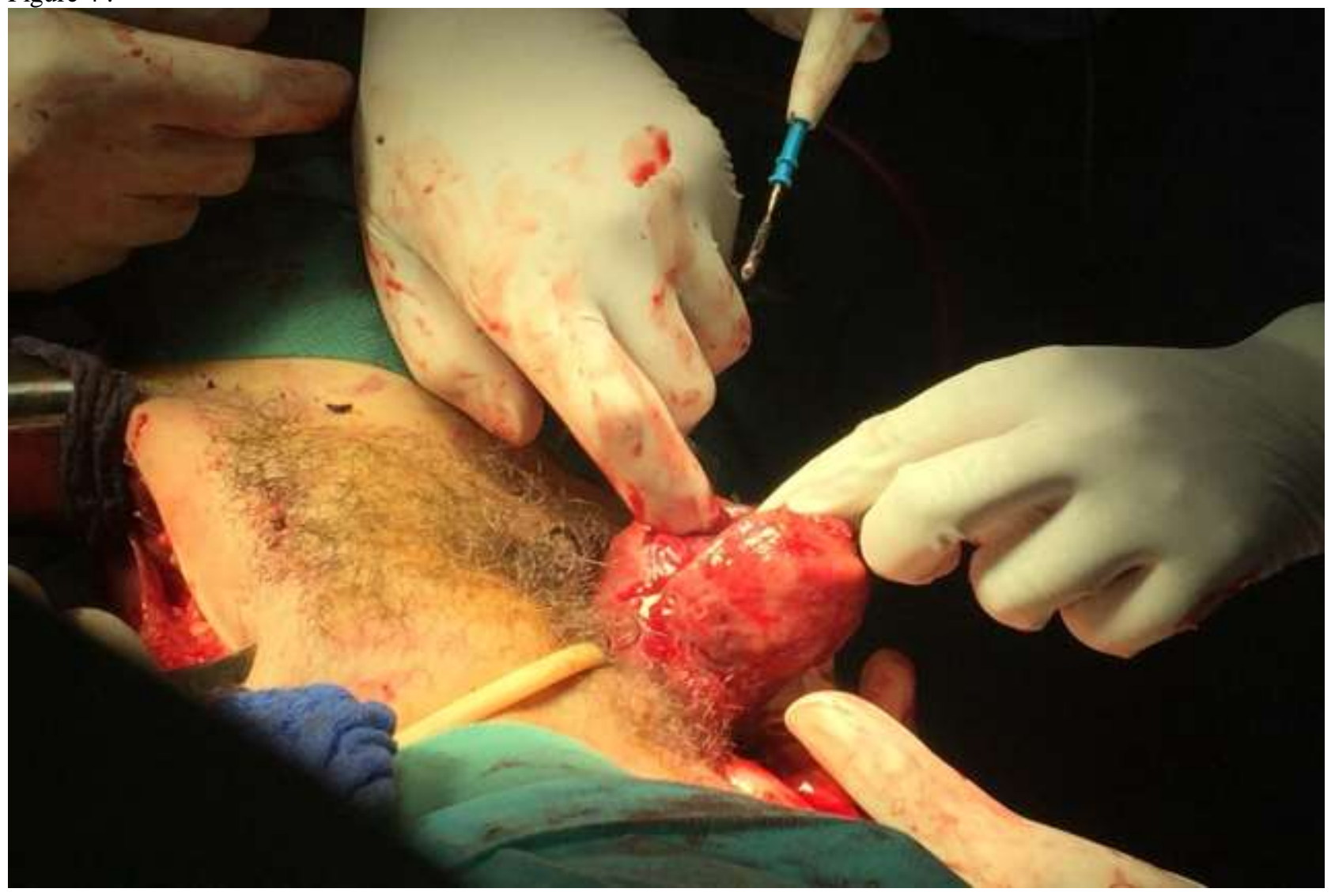

Figure 5 :

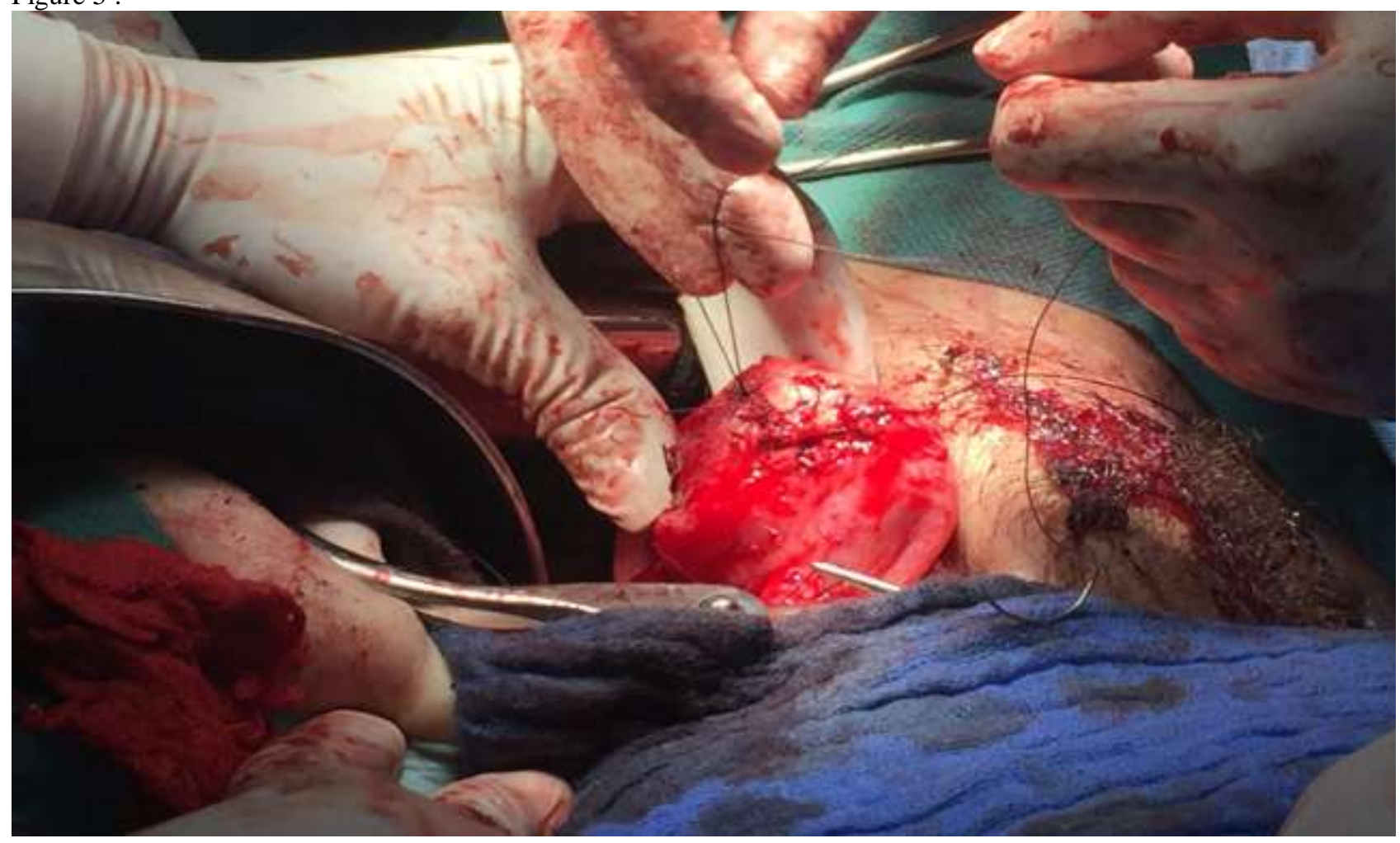




\section{Références:-}

1. Aspevik RK, Haffiner J Puerperal inversion of the uterus. Tidsskr Nor Laegeforen 1989;109:1403-4.

2. Salomon CG, Patek SK. Computed tomography of chronic nonpuerperal uterine inversion. J Comput Assist Tomogr 1990;14:1024-6.

3. Brahmi R, Ferhati D, Nabil S, et al. complete nonobstetrical uterine inversion. J Gynecol Obstet Biol Reprod 1995; 24:155-7.

4. Lai FM, Tseng P, Yeo SH, Tsakok FH. Nonpuerperal uterine inversion - a case report. Singapore Med J 1993;34:466-8.

5. Skinner GN, Louden KA. Nonpuerperal uterine inversion associated with an atypical leiomyoma. Aust N Z J Obstet Gynaecol 2001, 41: 100-1.

6. Takano K, Ichikawa Y, Tsunoda H, Nishida M. Uterine inversion caused by uterine sarcoma: a case report. Jpn J Clin Oncol 2001;31:39-42

7. Oguri H, Maeda N, Yamamoto Y, Wakatsuki A, Fukaya T. Non-Puerperal uterine inversion associated with endometrial carcinoma: a case report. Gynecol Oncol 2005;97:973-5

8. Moulding F, Hawnaur JM. MRI of non-puerperal uterine inversion due to endometrial carcinoma. Clin Radiol 2004;59:534-7

9. Lewin JS, Bryan PJ. MR imaging of uterine inversion. J Comput Assist Tomogr 1989;13:357-9

10. Gowri V. Uterine inversion and corpus malignancies: a historical review. Obstet Gynecol Surv 2000;55:703-7 